\title{
Avaliação do Desempenho Operacional do harvester 911.3 X3M em Áreas Declivosas
}

\author{
Renato Cesar Gonçalves Robert ${ }^{1}$, Flávia Alessandra Pinheiro Cordeiro e Silva ${ }^{2}$, \\ Marcio Pereira da Rocha ${ }^{1}$, Edvaldes José do Amaral $^{3}$, lvânio Luiz Guedes ${ }^{3}$
}

${ }^{1}$ Departamento de Engenharia e Tecnologia Florestal, Universidade Federal do Paraná - UFPR, Curitiba/PR, Brasil ${ }^{2}$ Curso de Engenharia Florestal, Universidade Federal do Paraná - UFPR, Curitiba/PR, Brasil ${ }^{3}$ Celulose Nipo Brasileira S.A, Ipatinga/MG, Brasil

\begin{abstract}
RESUMO
O presente trabalho teve como objetivo avaliar as operações de corte florestal do harvester 911.3 X3M em diferentes classes de declividade e sentidos de operação, bem como avaliar os movimentos nas distintas operações da máquina. Este harvester foi desenvolvido na Alemanha para atuar em declividades de até $40^{\circ}$ e ainda não havia sido utilizado em plantações florestais no Brasil. Os resultados evidenciaram que a produtividade não decresceu com o aumento da declividade, assim como os diferentes sentidos de operação não influenciaram no volume de madeira colhido por hora.
\end{abstract}

Palavras-chave: mecanização, declividade, corte florestal.

\section{Performance Evaluation of a Harvester 911.3 X3M Operating on Steep Terrain}

\begin{abstract}
This study aims to evaluate the performance of a harvester $911.3 \mathrm{X} 3 \mathrm{M}$ in harvesting operations at different slope terrain classes and directions (upward and downward), as well as the movements in the various machine operations. This harvester was developed in Germany to operate on slopes of up to $40^{\circ}$ and has not yet been used in forest plantations in Brazil. Results showed that the productivity did not decrease with increased slope, and upward and downward directions of operation did not influence the volume of timber harvested per hour.
\end{abstract}

Keywords: mechanization, steep terrain, harvesting operation.

\section{INTRODUÇÃO}

As operações de corte florestal em terrenos declivosos no Brasil geralmente são realizadas com um grande contingente de mão de obra, em que o método semimecanizado com uso da motosserra ainda predomina. Assim, as operações se resumem, em sua maioria, na derrubada das árvores e, em seguida, na sua extração, que geralmente é feita com tratores agrícolas adaptados com guinchos arrastadores; em casos menos frequentes, são usados guindastes e cabos aéreos, podendo também ser realizados, além da derrubada, o traçamento e o desgalhamento ainda no local de derrubada, sendo que essas operações são realizadas, na sua maioria, após a extração.

Tendo em vista as dificuldades impostas aos trabalhadores florestais em terrenos acidentados, Sant'Anna \& Malinovski (1999) apontaram que, em 
declividades variando de 20 a 75\%, o maior número de acidentes ocorreu na derrubada.

Com a necessidade de redução de custos operacionais, bem como da redução de mão de obra em algumas localidades, muitas empresas vêm adotando o emprego de modernas tecnologias para possibilitar a otimização de sistemas mecanizados de colheita de madeira (Yamashita, 2002). Nesse contexto, a mecanização florestal nas operações de corte causa a diminuição da carga de trabalho manual (Fenner, 1991) e, consequentemente, minimiza a ocorrência de acidentes e eventuais danos físicos aos trabalhadores.

Martins et al. (2009) concluíram que, dentre as ações compreendidas no ciclo do corte de árvores com harvester, o traçamento foi a que teve o maior tempo médio gasto dentro da operação e que o volume médio individual (VMI) foi a variável que mais explicou a capacidade operacional efetiva do harvester.

Em estudo realizado com o harvester marca Valmet modelo 911 X3M, Korten et al. (2003) apontaram que, em condições de trabalho e manejo realizado em florestas naturais da Alemanha, não se considerando a variação na declividade, a produtividade do harvester X3M ocupou 51\% do tempo de trabalho da máquina somente com as operações de corte e processamento.

Em um trabalho sobre o uso da combinação do harvester 911 X3M com o forwarder com cabo de tração, o Thuringian Ministry for Agriculture, Forestry, Environmentand Nature Conservation (2011) indica, como recomendação para seu melhor desempenho, que este sistema deve trabalhar em condições de floresta natural com árvores que possuam o diâmetro à altura do peito (DAP) médio em torno de $25 \mathrm{~cm} \mathrm{e}$ declividades variando de $35 \%$ a $50 \%$.

Assim, estudos sobre as variáveis que influenciam a produtividade na colheita florestal são imprescindíveis no planejamento da colheita, visando à redução de custos e ao melhor aproveitamento do sistema. O presente trabalho teve como objetivo analisar o desempenho do harvester da marca Komatsu Forest modelo 911.3X3M em diferentes classes de declividade e sentidos de operação, e corte com distintos comprimentos de tora, bem como avaliar o ciclo operacional da máquina em plantações de eucalipto.

\section{MATERIAL E MÉTODOS}

\section{1. Áreas de estudo}

A pesquisa foi realizada em áreas de uma empresa florestal, em talhões localizados nos municípios de Ferros e Antônio Dias, no Estado de Minas Gerais, Brasil. As áreas situam-se a $19^{\circ} 23^{\prime} 43^{\prime \prime}$ de latitude sul e $42^{\circ} 48^{\prime} 18^{\prime \prime} \mathrm{W}$ de longitude oeste. A temperatura média anual é de $19,9{ }^{\circ} \mathrm{C}$, com precipitação média de chuva anual de 1.292,6 mm. O tipo de clima característico da região é Cwa (Köppen) e a altitude média é de 640 metros acima do nível do mar.

$\mathrm{O}$ experimento foi conduzido em uma floresta de Eucalyptus spp. de primeiro corte, em talhões homogêneos e seminais, com idade variando entre 80 e 90 meses. O plantio possuía espaçamento de $3,00 \times 3,33$ metros, com declividades variando de $7^{\circ}$ a $38^{\circ}$.

De acordo com dados da empresa, que seguem a classificação apresentada pela Embrapa (1999), o tipo de solo predominante na região é o argilo-arenoso de cor castanho-avermelhado, com topografia caracterizada como montanhosa.

\subsection{Descrição da máquina}

O harvester analisado é composto por uma máquina de rodados de esteira adaptados em um boogie de pneus da marca Komatsu Forest, modelo 911.3 X3M, com motor diesel modelo 74 AWI e 228 HP. O peso operacional é de $25 \mathrm{t}$ e o alcance máximo da grua é $10 \mathrm{~m}$. Esta máquina foi desenvolvida para realizar as operações de corte florestal em áreas de declividade consideradas muito acentuadas, sendo equipada com um cabeçote Komatsu 360.2, com capacidade máxima de corte de $650 \mathrm{~mm}$, abertura máxima dos rolos de $550 \mathrm{~mm}$ e massa de $1.245 \mathrm{~kg}$. O sistema de controle eletrônico da máquina e do cabeçote é o Max Xplorer (Komatsu, 2012).

\subsection{Sistema de colheita}

De acordo com a classificação do sistema de colheita citada por Malinovski et al. (2006), o sistema 
de colheita utilizado foi o cut-to-length (toras curtas), em que o corte e o processamento das árvores eram realizados pelo harvester, perpendicularmente às curvas de nível e com eito de derrubada composto por quatro árvores, sendo a extração feita por forwarder.

\subsection{Coleta dos dados}

As coletas foram realizadas no período de novembro de 2011 a janeiro de 2012, durante o turno de trabalho compreendido entre 7 e 17 horas.

Os dados de tempos e movimentos foram obtidos pelo método de multimomento, com intervalo de 15 segundos, por observação. Conforme citado por Batista (2008), esse método trabalha com o cronômetro girando continuamente, com a determinação dos tempos das ações segundo a frequência com que estas ocorrem em um intervalo fixo.

Os valores referentes à produtividade $\left(\mathrm{m}^{3} \mathrm{~h} .{ }^{-1}\right)$ foram mensurados por meio do sistema de informação e controle da máquina (Max Xplorer). Foi calculada a intensidade amostral para todas as variáveis estudadas, de acordo com a Equação 1:

$n \geq \frac{t^{2} \cdot S^{2}}{E^{2}}$

em que: $\mathrm{n}=$ número de unidades amostrais necessárias; $\mathrm{t}=$ valor de " $t$ " de Student, para o nível de probabilidade desejado e (n-1) graus de liberdade; $S^{2}=$ variância; $\mathrm{E}=$ erro admissível (\%).

\subsection{Declividade}

$\mathrm{Na}$ avaliação da influência da declividade na produtividade do harvester $911.3 \mathrm{X} 3 \mathrm{M}$, a área experimental foi subdividida em parcelas, com 60 árvores em média por parcela (sendo 4 linhas e 15 árvores por linha), contemplando três classes de declividade: de 0 a $20^{\circ}$, de 20,1 a $27^{\circ}$ e acima de $27^{\circ}$. Na medição dos valores de declividades, foi utilizado um clinômetro eletrônico Haglöf, no modelo metros/graus. Nas parcelas amostradas, os comprimentos de tora variaram aleatoriamente, sendo de 2,6 m (com casca), 3,3 m (com casca), 6,0 $\mathrm{m}$ (com casca) e 6,3 m (sem casca). Entre os distintos comprimentos, incluíram-se árvores nas quais foi feito o descascamento e árvores com casca, sendo esta distribuição aleatória nas unidades amostradas.

O delineamento experimental utilizado foi em blocos ao acaso, com três tratamentos (classes de declividade) de diferentes números de parcelas e três blocos (comprimentos de tora: 3,3 m com casca, 6,0 $\mathrm{m}$ com casca e 6,3 m sem casca) para cada classe de declividade, totalizando 87 parcelas amostradas. O comprimento de 2,6 $\mathrm{m}$ não foi utilizado em função de a maioria das parcelas amostradas se localizar em uma só faixa de declividade.

O teste de Bartlett foi usado para verificar a homogeneidade das variâncias e foi feita a análise de variância a $95 \%$ de probabilidade. Realizou-se também a análise de regressão, a fim de estabelecer a relação entre a produtividade e a declividade.

\subsection{Sentido da operação}

Avaliou-se a produtividade do harvester enquanto o mesmo realizava as operações de corte movimentando-se em sentido ascendente e descendente. O número de parcelas foi de 70 , número acima da intensidade amostral recomendada para um erro de amostragem admissível de 5\% e um limite de erro de 10\%, que foi calculada em 29 amostras.

Para análise estatística dos dados, utilizouse o teste de significância com a distribuição do tipo " $t$ ", para médias de amostras independentes e com o mesmo número de observações, a 95\% de probabilidade.

\subsection{Avaliação de movimentos nas operações de corte e processamento}

Foi utilizada a marcação do tempo pelo método de multimomento, com observações em intervalos de 15 segundos durante o trabalho em cada parcela. $\mathrm{O}$ ciclo operacional foi estabelecido por meio do tempo de observação de operação parcial pelo número de árvores na parcela. Para definição da atividade do ciclo operacional do harvester que estava sendo realizada no momento de medição, foi considerado o momento exato na observação de cada ciclo. O ciclo operacional foi subdividido nas seguintes operações parciais: (1) Deslocamento da máquina: tempo consumido na movimentação da máquina até o início das atividades do cabeçote; (2) Posicionamento 
do cabeçote; (3) Corte; (4) Tombamento; (5) Desgalhamento; (6) Descascamento; (7) Passada do rolo: movimentação da tora no cabeçote; (8) Traçamento; (9) Empilhamento; (10) Descarte; (11) Recuperação: consiste no tempo gasto na recuperação de árvores que o cabeçote porventura não conseguiu segurar; (12) Apoio para pilha: tempo que a máquina utilizou para realizar os apoios para as pilhas nos eitos de derrubada, denominados de "travesseiros"; (13) Pausa técnica: tempo despendido na manutenção corretiva da máquina; (14) Pausa pessoal: tempo gasto nas atividades fisiológicas do operador.

As médias das ações parciais nos distintos comprimentos de tora e sentido de operação foram avaliadas estatisticamente por meio do teste de " $t$ " para médias de amostras independentes com números diferentes de observações, para 95\% de probabilidade.

\section{RESULTADOS E DISCUSSÃO}

\subsection{Declividade}

A análise dos dados evidenciou que a produtividade do harvester não decresceu com o aumento do percentual de inclinação do terreno nas parcelas, resultando em uma produtividade média de $27,5 \mathrm{~m}^{3} \mathrm{~h}^{-1}$. Este valor se diferencia do valor médio de operação padrão da empresa em condições de $0^{\circ}$ a $24^{\circ}$ de declividade, que é de $21,8 \mathrm{~m}^{3} \mathrm{~h}^{-1}$. Esta diferença entre a produtividade média padrão da empresa e a produtividade média nas parcelas pode ser explicada pelo fato de que, na operação padrão, são levadas em consideração as operações de retorno dentro do eito sem realização da operação. Os volumes médios obtidos pelo sistema eletrônico da máquina, para as distintas classes de declividade nas parcelas amostradas, foram os seguintes: Classe I $\left(20^{\circ} \geq \mathrm{d} \geq 0^{\circ}\right): 26,5 \mathrm{~m}^{3} \mathrm{~h}^{-1}$; Classe II $\left(27^{\circ} \geq \mathrm{d} \geq 20,1^{\circ}\right)$ : $28,5 \mathrm{~m}^{3} \mathrm{~h}^{-1}$ e Classe III $\left(\mathrm{d}>27^{\circ}\right): 27,3 \mathrm{~m}^{3} \mathrm{~h}^{-1}$.

A Análise de Variância evidenciou que estatisticamente a produtividade não se diferenciou nas três classes de declividade. Esses resultados diferem dos obtidos por Simões \& Fenner (2010), que apresentaram diferenças de produtividade ao estudar a influência do relevo na produtividade de um harvester em plantações de eucalipto com declividades que variaram de 10 a 25\%. No entanto, além de as declividades do trabalho citado serem notadamente inferiores às adotadas no presente estudo, o harvester X3M foi projetado para trabalhar em altas declividades. A Tabela 1 apresenta a análise de variância da produtividade média em função das classes de declividade.

A Figura 1 representa os valores médios de volume $\left(\mathrm{m}^{3} \mathrm{~h}^{-1}\right)$ para as declividades medidas. A análise de regressão linear foi feita pelo método da Soma de Quadrados dos Produtos Corrigidos e demonstrou baixa correlação da produtividade do harvester com a declividade, com $\mathrm{R}=0,2332$ e erro padrão da estimativa de 22,2\%. Esta análise corrobora o resultado obtido com a análise de variância.

A baixa correlação da produtividade com a declividade difere dos resultados apresentados por Bramucci \& Seixas, 2002, Jiroušek et al., 2007, Martins et al., 2009 e Simões \& Fenner, 2010, que avaliaram a influência da declividade na produtividade de harvesters convencionais e não em harvesters especificamente projetados para operação em terrenos declivosos. O VMI pode ser, portanto, o fator que mais influenciou as diferentes produtividades, visto que Burla (2008) e Stampfer \& Steinmüller $(2004,2001)$ atestam que o volume médio individual é o principal fator de influência da produtividade de um harvester.

Fernandes et al. (2013), no estudo com harvester com rodado de pneus equipados com semiesteiras,

Tabela 1. Análise de variância da produtividade média em função das classes de declividade.

Table 1. Analysis of variance of mean productivity according to slope classes.

\begin{tabular}{lccccc}
\multicolumn{1}{c}{ Fonte de variação } & GL & SQ & QM & F & p \\
Blocos & 2 & 5,221 & 2,610 & 0,119 & $<0,005$ \\
Classes de declividade & 2 & 9,731 & 4,865 & 0,222 & \\
Resíduo & 4 & 87,685 & 21,921 & & \\
Total & 8 & 102,637 & & & \\
\hline
\end{tabular}




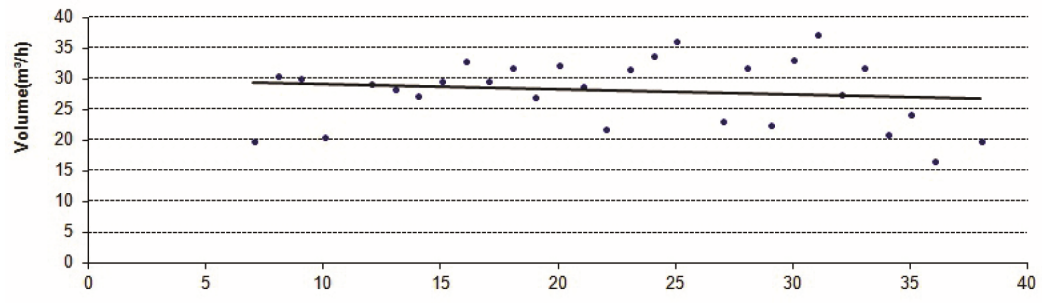

Declividade (graus)

EQ: $\operatorname{Vol}\left(\mathrm{m}^{3} \cdot \mathrm{h}^{-1}\right)=29,5021+0,0314$,decl (graus)

$R^{2}=0,2332$

Figura 1. Produtividade do harvester nas parcelas em diferentes declividades.

Figure 1. Harvester productivity in plots with different slope classes.

encontraram em declividades entre $20,1^{\circ}$ e $30^{\circ}$ as menores produtividades, fato este que se diferencia dos encontrados neste estudo. Portanto, a adaptação estrutural de esteiras no boogie do harvester 911.3 $\mathrm{X} 3 \mathrm{M}$ pode ser o principal motivo para que não exista diferença de produtividade em diferentes classes de declividade.

\subsection{Sentido de deslocamento}

O teste de " $\mathrm{t}$ " indicou que o sentido de deslocamento do harvester nas operações de corte nas parcelas não influenciou estatisticamente na produtividade. O volume médio no greide ascendente foi de $29,9 \mathrm{~m}^{3} \mathrm{~h}^{-1}$, enquanto no greide descendente foi de $27,5 \mathrm{~m}^{3} \mathrm{~h}^{-1}$. Essa diferença pode ser explicada em função da maior facilidade, segurança e ergonomia nas operações de corte no sentido ascendente (Stampfer \& Steinmüller, 2004).

A declividade média nos diferentes sentidos foi de $19^{\circ}$ e $22^{\circ}$, para os sentidos ascendente e descendente, respectivamente. $\mathrm{O}$ volume médio individual (VMI), quando a máquina operou na subida, foi de $0,243 \mathrm{~m}^{3}$, sendo que, na descida, esse volume foi de $0,230 \mathrm{~m}^{3}$.

Os resultados mostram que a derrubada e o processamento por um harvester em terrenos acidentados podem ocorrer quando os mesmos operam descendo e ascendendo, sendo que a operação tende a se tornar mais confortável e segura no sentido ascendente em razão de uma maior estabilidade da máquina. Nota-se que ocorre o inverso nas operações no sentido descendente, principalmente no que tange à estabilidade da máquina, quando a mesma derruba e processa com o braço mecânico e o cabeçote muito abaixo do nível da cabine da máquina.

\subsection{Estudo de tempos e movimentos}

A constituição média das atividades parciais do ciclo operacional do harvester está apresentada na Figura 2. Conforme pode ser visto, para todas as classes de declividade avaliadas, o maior tempo dispendido foi na operação de traçamento, com média de $24 \%$ do tempo total do ciclo operacional. Os percentuais de tempo nas distintas etapas do processo podem ser considerados similares nas diferentes declividades, o que corrobora os resultados obtidos na análise de produtividade em função das distintas classes de declividade.

O tempo gasto para as pausas operacionais (pausa técnica) foi, em média, 0,09\%, o que pode ser considerado muito baixo. Simões \& Fenner (2010) encontraram um valor médio de $13,6 \%$ do tempo total do ciclo operacional, enquanto Burla (2008) encontrou, para a mesma operação, valor médio de $1 \%$. Um aumento do percentual de tempo gasto na manutenção corretiva representa diminuição da produtividade, uma vez que aumenta o tempo do ciclo. Isto se deve ao fato de que a máquina em teste, antes de chegar ao Brasil, já possuía cerca de nove mil horas de uso, representando cerca de um terço da vida útil do harvester.

Spinelli et al. (2002), ao estudar a produtividade do harvester em terreno plano e declivoso, com processamento em toras de $2 \mathrm{~m}$ e $4 \mathrm{~m}$, concluíram que a as condições do terreno, o layout operacional, o volume e a forma das árvores, e o comprimento 
das toras influenciam na duração de cada operação parcial do harvester, mostrando assim que as diferenças dos tempos gastos encontradas em cada classe de declividade podem ter sido influenciadas pelo terreno.

As ações do ciclo operacional do harvester também foram analisadas separadamente no que concerne ao sentido de operação. As distintas operações parciais do ciclo podem ser analisadas na Figura 3, na qual se percebe que não há grande disparidade entre os valores dos dois sentidos de deslocamento. A ação de traçamento no sentido ascendente apresentou um valor $4,3 \%$ maior quando comparada a essa ação no sentido descendente.

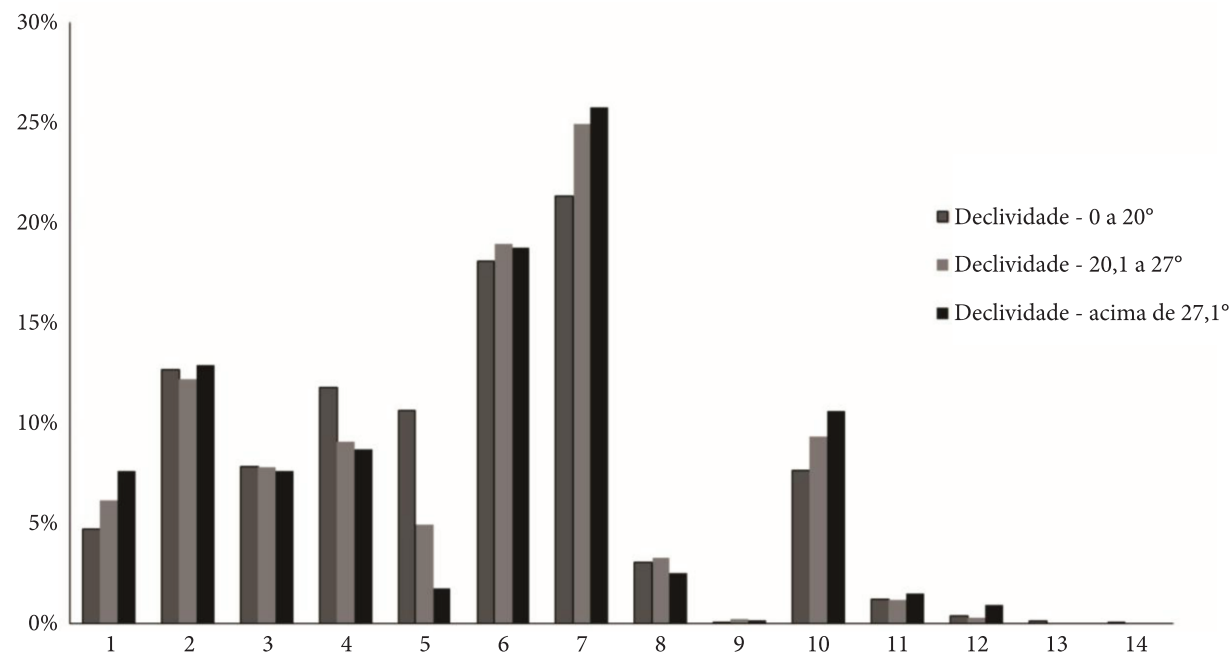

Figura 2. Percentuais de tempos das atividades parciais do harvester X3M em três classes de declividade, em que: (1)Deslocamento da máquina; (2)- Posicionamento do cabeçote; (3)- Corte; (4)- Tombamento; (5)- Descascamento; (6)- Processamento ou passada do rolo; (7)- Traçamento; (8)- Desgalhamento; (9)- Empilhamento; (10)- Descarte; (11)- Recuperação; (12)- Apoio para pilha; (13)- Pausa técnica; (14)- Pausa pessoal.

Figure 2. Time percentage of partial activities of harvester X3M in three slope classes, where: (1)- Machine displacement; (2)- Harvester head positioning; (3)- Tree Cut down; (4)- Tipping; (5)- Debarking; (6)- Harvester head scrolling; (7)- Log sectioning; (8) - Delimbing; (9)- Stacking; (10)- Discard; (11)- Recuperation; (12)Stack support; (13)- Technical pause; (14)Personal pause.

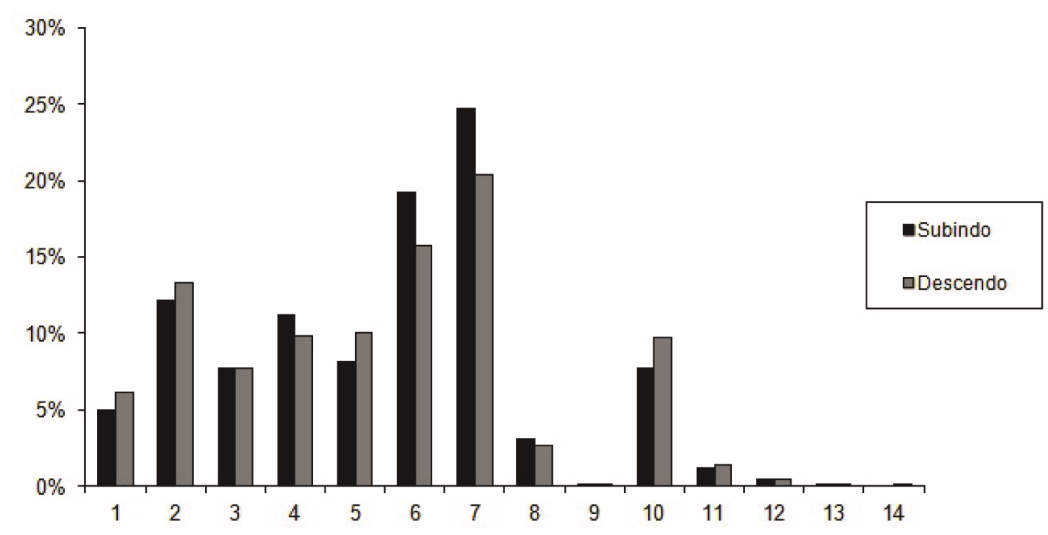

Figura 3. Percentuais de tempos das operaçõesparciais do harvester X3M nos sentidos de operação, em que: (1)Deslocamento da máquina; (2)- Posicionamento do cabeçote; (3)- Corte; (4)- Tombamento; (5)- Descascamento; (6)- Processamento ou passada do rolo; (7)- Traçamento; (8)- Desgalhamento; (9)- Empilhamento; (10)- Descarte; (11)- Recuperação; (12)- Apoio para pilha; (13)- Pausa técnica; (14)- Pausa pessoal.

Figure 3. Time percentage of partial operations of harvester X3M in upward and downward directions, where: (1)- Machine displacement; (2)- Harvester head positioning; (3)- Tree Cut down; (4)- Tipping; (5)- Debarking; (6)Harvester head scrolling; (7)- Log sectioning; (8) - Delimbing; (9)- Stacking; (10)- Discard; (11)- Recuperation; (12) Stack support; (13)- Technical pause; (14)Personal pause. 
De acordo com Frutig et al. (2007), o rendimento operacional do harvester em áreas declivosas está fortemente relacionado com a existência ou não de uma rede viária que garanta a otimização operacional desta máquina no seu melhor sentido de trabalho, uma vez que a existência de uma área - que geralmente é uma estrada - que permita a manobra do harvester quando o mesmo termina o corte de uma linha na parte mais baixa do terreno é de fundamental importância para o melhor aproveitamento da operação. Se essa área para manobra da máquina inexiste, o mesmo acaba voltando de ré para iniciar o corte; no caso de plantações florestais, esse início de corte se dará a partir da próxima linha na parte mais alta do terreno, prejudicando o rendimento operacional do processo.

\section{CONCLUSÕES}

Em todas as classes de declividades avaliadas, a produtividade do harvester não apresentou diferenças estatísticas significativas, apresentando um volume médio nas parcelas de $27,4 \mathrm{~m}^{3} \mathrm{~h}^{-1}$; indicase, dessa forma, a adequação deste modelo para a operação em terrenos acidentados.

O sentido de deslocamento do harvester nas operações de corte não influenciou a produtividade, possibilitando um ganho em relação ao método atualmente empregado, de retorno dentro do eito sem realização do corte.

A ação do ciclo operacional que mais demanda tempo na máquina estudada é o traçamento, que ocupou em média $24 \%$ do tempo total do ciclo.

\section{AGRADECIMENTOS}

Os autores agradecem às empresas CENIBRA $\mathrm{e}$ KOMATSU FOREST pela abertura e disponibilização de dados para esta pesquisa.

\section{STATUS DA SUBMISSÃO}

Recebido: 19/07/2012

Aceito: $27 / 04 / 2013$

Publicado: 30/06/2013
AUTOR(ES) PARA CORRESPONDÊNCIA

\section{Renato Cesar Gonçalves Robert}

Universidade Federal do Paraná,

Rua Lothário Meissner, 900,

CEP 80210-170, Curitiba, PR, Brasil

e-mail: renatorobert@ufpr.br

\section{REFERENNCIAS}

Batista HLP. Estudo de tempo e rendimento da motosserra considerando fatores ergonômicos numa exploração florestal na Amazônia Central [monografia]. Manaus: Instituto Nacional de Pesquisas da Amazônia - INPA; 2008.

Bramucci M, Seixas F. Determinação equantificação de fatores deinfluência sobrea produtividadede "harvesters" na colheita florestal. ScientiaForestalis 2002; 62: 62-74.

Burla ER. Avaliação técnica e econômica do harvester na colheita do eucalipto [dissertação]. Viçosa: Universidade Federal de Viçosa; 2008.

Embrapa. Serviço Nacional de Levantamentos de Solo: Sistema Brasileiro de Classificação de Solos. Brasília: Embrapa, Produção da Informação; Rio de Janeiro: Embrapa Solos; 1999. 412 p.

Fenner, PT. Estudo descritivo dos acidentes de trabalho em uma empresa florestal [dissertação]. Curitiba: Universidade Federal do Paraná; 1991.

Fernandes HC, Burla ER, Leite ES, Minette LJ. Avaliação técnica e econômica de um "Harvester" em diferentes condições de terreno e produtividade da Floresta. ScientiaForestalis 2013; 41(97): 141-151.

Frutig F, Fahrni F, Stettler A, Egger A. Mechanisierte Holzernte in Hanglagen. Wald und Holz 2007; 88(4): 4752.

Jiroušek R, Klvač R, Skoupý A. Productivityand costs of the mechanized cut-to-length wood harvesting system in clear-felling operations. Journal of Forest Science 2007; 53(10): 476-482.

Komatsu Forest. 911.5 X3M. [cited 2012 abr. 25]. Available from: http://www.komatsuforest.com.br/ default.aspx?id=64347\&productId $=54344$.

Korten S, Matthies D, Gennari C, Nagl M. Leistung, Kosten, Pfleglichkeit des Valmet 911 X3M. Holzernte am Hang, Forst \& Technik; 2003

Malinovski JR, Malinovski RA, Camargo CMS. Sistemas. In: Machado CC, editor. Colheita Florestal. Viçosa: Universidade Federal de Viçosa, Imprensa Universitária; 2006.

Martins RJ, Seixas F, Stape JL. Avaliação técnica e econômica de um harvester trabalhando 
em diferentes condições de espaçamento e arranjo de plantio em povoamento de eucalipto. ScientiaForestalis 2009, 37(83): 253-263

Sant'Anna CM, Malinovski JR. Avaliação da segurança no trabalho de operadores de motosserra no corte de eucalipto em região montanhosa. Ciência Florestal 1999; 9(2): 75-84.

Simões D, Fenner PT. Influência do relevo na produtividade e custos do harvester. ScientiaForestalis 2010, 38(85): 107-114.

Spinelli R, Owende PMO, Ward SM. Productivity and cost of CTL harvesting of Eucalyptus globulus stands using excavator - based harvesters. Forest Products Journal 2002; 52(1): 67-57.

Stampfer K, Steinmüller T. A New Approach To Derive A Productivity Model for the Harvester "Valmet 911 Snake". In: Anais do The International Mountain Logging and 11th Pacific Northwest Skyline Symposium; 2001. p. 254-262. [cited 2012 sep. 01]. Available from: http:// depts.washington.edu/sky2001/proceedings/papers/ Stampfer.pdf.

Stampfer K, Steinmüller T. Leistungsdaten Valmet 911.1 X3 $M$ - Endbericht. Universität für Bodenkultur Wien - Department für Wald- und Boden-Wissenschaft; Nov 2004.

Thuringian Ministry For Agriculture, Forestry, Environment And Nature Conservation (DE). Timber harvesting methods in steep and moderately sloped terrain. Erfurt. Fachhochschule Erfurt: University of Applied Sciences - Forstwissenschaft und Ökosystemmanagement; 2011. [CD-ROM].

Yamashita RY. Avaliação das condições de trabalho e da exposição à vibração do operador de máquinas na colheita florestal [dissertação]. Piracicaba: Escola Superior de Agronomia Luis de Queiroz; 2002. 